\title{
Modified Dust-Lower-Hybrid Waves In Quantum Plasma
}

\author{
Abdul Rauf ${ }^{1}$, I. Zeba ${ }^{2}$ and Muhammad Saqlain ${ }^{3}$ \\ ${ }^{1}$ Department of Physics, LGU, Lahore, Pakistan \\ ${ }^{2}$ Department of Physics, LCW University Lahore-54000, Pakistan \\ ${ }^{3}$ Department of Mathematics, Lahore Garrison University, Lahore, Pakistan
}

\begin{abstract}
Dust-lower-hybrid waves in quantum plasma have been studied. The dispersion relation of the dust-lower-hybrid wave has been examined using the quantum hydrodynamic model of plasma in an ultra-cold Fermi dusty plasma in the presence of a uniform external magnetic field. Graphical analysis shows that the electron Fermi temperature effect and the quantum corrections give rise to significant effects on the dust-lower-hybrid wave of the magnetized quantum dusty plasma.
\end{abstract}

Keywords: Magneto plasma, wavelength, microecectronics, hydrodynamics, quantum mechanics

\section{Introduction}

Quantum mechanical effects in some specific areas of plasma physics have great significance. When plasma is cooled to an extremely low temperature, the de Broglie wavelengths of the plasma particles are comparable to the dimensions of the system. In such plasmas, the ultra-cold dense plasma would behave as a Fermi gas and quantum mechanical effects might play a vital role in the behaviour of the charge carriers of plasmas under these conditions. In microelectronics [1,2] and very large integrated circuit fabrications, the system may develop contaminants due to etching, implantations etc. which may lead to new properties. The laser produced plasmas [3] and plasmas in high density astrophysical objects [4-6] may also be contaminated by various reasons. Thus, these ultra-cold plasma systems may behave as dusty plasmas where quantum mechanical effects could unveil the properties of these systems. This research aims to present the quantum effects on dust-lower-hybrid waves, where dust dynamic plays a vital role. Within the ambit of a symmetrical external magnetic field, the modification of the dust-lower-hybrid wave in quantum plasma can be achieved by following the standard techniques [7-10].

\section{Material and Method}

The equations in the quantum hydrodynamic model for electrons and ions of dusty plasma in presence of a dust-lower-hybrid perturbation and $\vec{B}_{0}$ are

$$
\frac{\partial n_{j 1}}{\partial t}+n_{j o}\left(\vec{\nabla} \cdot \vec{V}_{j 1}\right)=0
$$

$n_{j 1}=$ perturb particle density of $j$ th species

$n_{j o}=$ equilibrium particle density of $\mathrm{jth}$ species

$\vec{V}_{j 1}=$ perturb velocity of jth species

The equation of motion for electron and ions of dusty plasma in the presence of perturbation and $\vec{B}_{0}$ is 


$$
m_{j} n_{j o} \frac{\partial \vec{V}_{j 1}}{\partial t}=n_{j o} q_{j}\left(\vec{E}_{1}+\vec{V}_{j 1} \times \vec{B}_{o}\right)
$$

$m_{j}=$ mass of $\mathrm{jth}$ species

$q_{j}=$ charge of $j$ th species

$\vec{E}_{1}=$ electric field intensity in the presence of a DLH perturbation

$\vec{B}_{0}=$ equilibrium/uniform magnetic field.

$\varphi_{1}=$ perturb electrostatic potential

$$
\vec{E}_{1}=-\vec{\nabla} \varphi_{1}
$$

and

So equation (2) becomes

$$
\frac{q_{j} \vec{B}_{0}}{m_{j}}=\vec{\omega}_{c j}
$$

$$
\frac{\partial}{\partial t} \vec{V}_{j 1}=-\frac{q_{j}}{m_{j}} \vec{\nabla} \varphi_{1}+\vec{V}_{j 1} \times \vec{\omega}_{c j}
$$

Dielectric susceptibility of plasma can be obtained by solving the equations (1) and (3)

according to Fourier Laplace transformation

From equation (3)

$$
\begin{aligned}
& \frac{\partial}{\partial t}=-i \omega \quad \text { and } \quad \vec{\nabla}=i \vec{k} \\
& \vec{V}_{j 1}=\vec{V}_{\perp}+\vec{V}_{\|} \\
& \vec{k}=\vec{k}_{\perp}+\vec{k}_{\|}
\end{aligned}
$$

$$
\vec{V}_{\perp}=\frac{i q_{j} \varphi_{1}\left[\left(\vec{k}_{\perp} \times \vec{\omega}_{c j}\right)-i \vec{k}_{\perp} \omega\right]}{m_{j}\left(\omega^{2}-\omega_{c j}^{2}\right)}
$$

And

From equation (1)

$$
\vec{V}_{\|}=\frac{q_{j} \varphi_{1} \vec{k}_{\|}}{\omega m_{j}}
$$

$$
\begin{array}{r}
n_{j 1}=\frac{n_{j o}}{\omega}\left[\vec{k}_{\perp} \cdot \vec{V}_{\perp}+\vec{k}_{\|} \cdot \vec{V}_{\|}\right] \\
n_{j 1}=\frac{n_{j o}}{\omega}\left[\frac{k_{\perp}^{2} \omega q_{j} \varphi_{1}}{m_{j}\left(\omega^{2}-\omega_{c j}^{2}\right)}+\frac{k_{\|}^{2} q_{j} \varphi_{1}}{\omega m_{j}}\right]
\end{array}
$$

As

$$
n_{j 1}=-\frac{\chi_{j} k^{2} \varphi_{1}}{4 \pi q_{j}}
$$

From equations (4) and (5)

Since

$$
\chi_{j}=\frac{k_{\perp}^{2}}{k^{2}} \times \frac{4 \pi n_{j o} q_{j}^{2}}{m_{j}\left(\omega^{2}-\omega_{c j}^{2}\right)}-\frac{k_{\|}^{2}}{k^{2}} \times \frac{4 \pi n_{j o} q_{j}^{2}}{\omega^{2} m_{j}}
$$

$$
\omega_{p j}^{2}=\frac{4 \pi n_{j o} q_{j}^{2}}{m_{j}}
$$


Therefore

$$
\chi_{j}=\frac{k_{\perp}^{2}}{k^{2}} \times \frac{\omega_{p j}^{2}}{\left(\omega_{c j}^{2}-\omega^{2}\right)}-\frac{k_{\|}^{2}}{k^{2}} \times \frac{\omega_{p j}^{2}}{\omega^{2}}
$$

For un-magnetized dust

$$
\begin{gathered}
\omega_{c d}=0 \\
\chi_{d}=-\frac{k_{\perp}^{2}}{k^{2}} \times \frac{\omega_{p d}^{2}}{\omega^{2}}-\frac{k_{\|}^{2}}{k^{2}} \times \frac{\omega_{p d}^{2}}{\omega^{2}}
\end{gathered}
$$

So, the susceptibility for the unmagnetized and cold dust particle is

The dispersion relation is

$$
\chi_{d}=-\frac{\omega_{p d}^{2}}{\omega^{2}}
$$

$$
\begin{gathered}
\varepsilon(\omega, \vec{k})=\varepsilon=1+\chi_{e}+\chi_{i}+\chi_{d}=0 \\
1+\left(\frac{k_{\perp}^{2}}{k^{2}} \times \frac{\omega_{p e}^{2}}{\left(\omega_{c e}^{2}-\omega^{2}\right)}-\frac{k_{\|}^{2}}{k^{2}} \times \frac{\omega_{p e}^{2}}{\omega^{2}}\right) \\
+\left(\frac{k_{\perp}^{2}}{k^{2}} \times \frac{\omega_{p i}^{2}}{\left(\omega_{c i}^{2}-\omega^{2}\right)}-\frac{k_{\|}^{2}}{k^{2}} \times \frac{\omega_{p i}^{2}}{\omega^{2}}\right)+\left(-\frac{\omega_{p d}^{2}}{\omega^{2}}\right)=0 \\
\omega_{c e}^{2} \gg \omega^{2} \text { and } \omega_{c i}^{2} \gg \omega^{2}
\end{gathered}
$$

Also

$$
\begin{gathered}
\omega_{p e} \gg \omega_{p i} \\
\omega_{p i}^{2} \approx 0 \\
\omega_{p i}^{2} \gg \omega_{c i}^{2} \\
\omega^{2}=\frac{k_{\perp}^{2}}{k^{2}} \times \frac{\omega_{p d}^{2} \omega_{c i}^{2}}{\omega_{p i}^{2}}\left(1+\frac{k_{\|}^{2}}{k^{2}} \frac{\omega_{p e}^{2}}{\omega_{p d}^{2}}\right)
\end{gathered}
$$

As, $\quad k^{2}=k_{\perp}^{2}+k_{\|}^{2} \quad$ if $\quad k_{\perp}^{2} \gg k_{\|}^{2} \quad$ then, $\quad \boldsymbol{k}^{2}=\boldsymbol{k}_{\perp}^{2} \quad$ or $\quad \frac{\boldsymbol{k}^{2}}{\boldsymbol{k}_{\perp}^{2}}=\mathbf{1}$

$$
\begin{gathered}
\omega^{2}=\frac{\omega_{p d}^{2} \omega_{c i}^{2}}{\omega_{p i}^{2}}\left(1+\frac{k_{\|}^{2}}{k^{2}} \frac{\omega_{p e}^{2}}{\omega_{p d}^{2}}\right) \\
\omega^{2}=\omega_{d l h}^{2}\left(1+\frac{k_{\|}^{2}}{k^{2}} \frac{\omega_{p e}^{2}}{\omega_{p d}^{2}}\right)
\end{gathered}
$$

is the dispersion relation for DLH wave.

Now, equation (2) with quantum effect becomes

$$
\boldsymbol{m}_{j} \boldsymbol{n}_{j o} \frac{\partial \vec{V}_{j 1}}{\partial t}=n_{j o} \boldsymbol{q}_{j}\left(\vec{E}_{1}+\vec{V}_{j 1} \times \vec{B}_{o}\right)-\vec{\nabla} P_{j 1}+\frac{\hbar^{2} \vec{\nabla}\left(\nabla^{2} n_{j 1}\right)}{4 m_{j}}
$$

$\frac{\hbar^{2} \vec{\nabla}\left(\nabla^{2} n_{j 1}\right)}{m_{j}}=$ Bohm Potential term

$\vec{\nabla} P_{j 1}=$ Fermi pressure term

where

$$
\frac{\partial \vec{V}_{j 1}}{\partial t}=\frac{q_{j}}{m_{j}}\left(-\vec{\nabla} \varphi_{1}+\vec{V}_{j 1} \times \vec{B}_{o}\right)-\frac{\vec{\nabla} P_{j 1}}{m_{j} n_{j o}}+\frac{\hbar^{2} \vec{\nabla}\left(\nabla^{2} n_{j 1}\right)}{4 m_{j}^{2} n_{j o}}
$$

$$
P_{j 1}=2 n_{j 1} k_{B} T_{F j}
$$

- by assuming that electron and ions possess significant quantum mechanical effects 
- by neglecting the quantum effects on dusty particles (because of high mass which gives to an insignificant de Broglie wavelength).

Since

$$
\begin{gathered}
\frac{\partial}{\partial t}=-i \omega \text { and } \vec{\nabla}=i \vec{k} ; \quad \nabla^{2}=(i k)^{2}=-k^{2} \\
-i \omega \vec{V}_{j 1}=-\frac{q_{j}}{m_{j}} i \vec{k} \varphi_{1}+\frac{q_{j}}{m_{j}}\left(\vec{V}_{j 1} \times \vec{B}_{o}\right)-\frac{i \vec{k}\left(2 n_{j 1} k_{B} T_{F j}\right)}{m_{j} n_{j o}} \\
+\frac{\hbar^{2}(i \vec{k})\left(-k^{2} n_{j 1}\right)}{4 m_{j}^{2} n_{j o}} \\
\omega \vec{V}_{j 1}=\frac{q_{j}}{m_{j}} \vec{k} \varphi_{1}+i \frac{q_{j}}{m_{j}}\left(\vec{V}_{j 1} \times \vec{B}_{o}\right)+\frac{2 \vec{k}}{n_{j o}}\left(\frac{k_{B} T_{F j}}{m_{j}}\right) n_{j 1}+\frac{\hbar^{2}(\vec{k})\left(k^{2}\right)}{4 m_{j}^{2} n_{j o}} n_{j 1} \\
\omega \vec{V}_{j 1}=\frac{q_{j}}{m_{j}} \vec{k} \varphi_{1}+i \frac{q_{j}}{m_{j}}\left(\vec{V}_{j 1} \times \vec{B}_{o}\right)+\frac{V_{F j}}{n_{j o}}\left(1+\gamma_{j}\right) n_{j 1}
\end{gathered}
$$

where

where

$$
\begin{array}{r}
V_{F j}=\left(\frac{2 k_{B} T_{F j}}{m_{j}}\right)^{\frac{1}{2}} \text { and } \gamma_{j} \\
=\frac{\hbar^{2}\left(k^{2}\right)}{8 m_{j} k_{B} T_{F j}}(\text { Quantum correction }) \\
\omega \vec{V}_{j 1}=\frac{q_{j}}{m_{j}} \vec{k} \varphi_{1}+i\left(\vec{V}_{j 1} \times \vec{\omega}_{c j}\right)+\frac{\vec{k} V_{F j}^{2}}{n_{j o}} n_{j 1}
\end{array}
$$

From equation (1)

$$
V_{F j}^{\prime 2}=V_{F j}^{2}\left(1+\gamma_{j}\right)
$$

$$
\begin{gathered}
-i \omega n_{j 1}+n_{j o}\left(\overrightarrow{i k} \cdot \vec{V}_{j 1}\right)=0 \\
-\omega n_{j 1}+n_{j o}\left(\vec{k} \cdot \vec{V}_{j 1}\right)=0 \\
n_{j 1}=\frac{n_{j o}}{\omega} \vec{k} \cdot \vec{V}_{j 1}
\end{gathered}
$$

So equation (12) becomes

where

$$
\begin{gathered}
\omega \vec{V}_{j 1}=\frac{q_{j}}{m_{j}} \vec{k} \varphi_{1}+i\left(\vec{V}_{j 1} \times \vec{\omega}_{c j}\right)+\frac{\vec{k} V_{F j}^{\prime 2}}{n_{j o}}\left(\frac{n_{j o}}{\omega} \vec{k} \cdot \vec{V}_{j 1}\right) \\
\omega \vec{V}_{j 1}=\frac{q_{j}}{m_{j}} \vec{k} \varphi_{1}+i\left(\vec{V}_{j 1} \times \vec{\omega}_{c j}\right)+\frac{k^{2} V_{F j}^{\prime 2}}{\omega}\left(\vec{V}_{j 1}\right) \\
\omega\left(1-\frac{k^{2} V_{F j}^{\prime 2}}{\omega^{2}}\right) \vec{V}_{j 1}=\frac{q_{j}}{m_{j}} \vec{k} \varphi_{1}+i\left(\vec{V}_{j 1} \times \vec{\omega}_{c j}\right)
\end{gathered}
$$

$$
\begin{gathered}
F_{j}^{\prime}=1-\frac{k^{2} V_{F j}^{\prime 2}}{\omega^{2}} \\
\omega F_{j}^{\prime}\left(\vec{V}_{j 1}\right)=\frac{q_{j}}{m_{j}} \vec{k} \varphi_{1}+i\left(\vec{V}_{j 1} \times \vec{\omega}_{c j}\right)
\end{gathered}
$$


Taking perpendicular $(\perp)$ component of equation (14)

$$
\boldsymbol{\omega} \boldsymbol{F}_{j}^{\prime}\left(\vec{V}_{j \perp}\right)=\frac{q_{j}}{m_{j}} \vec{k}_{\perp} \varphi_{1}+\boldsymbol{i}\left(\vec{V}_{j \perp} \times \overrightarrow{\boldsymbol{\omega}}_{c j}\right)
$$

Multiply this equation by $\overrightarrow{\boldsymbol{\omega}}_{\boldsymbol{c} j}$, we get

$$
\begin{aligned}
& \omega F_{j}^{\prime}\left(\vec{V}_{j \perp} \times \vec{\omega}_{c j}\right)=\frac{q_{j} \varphi_{1}}{m_{j}}\left(\vec{k}_{\perp} \times \vec{\omega}_{c j}\right)+i\left(\vec{V}_{j \perp} \times \vec{\omega}_{c j}\right) \times \vec{\omega}_{c j} \\
& -i \omega F^{\prime}{ }_{j}\left(-i \omega F^{\prime}{ }_{j} \vec{V}_{j \perp}+\frac{i q_{j} \vec{k}_{\perp} \varphi_{1}}{m_{j}}\right)=-i \frac{q_{j} \varphi_{1}}{m_{j}}\left(\vec{k}_{\perp} \times \vec{\omega}_{c j}\right)+ \\
& \left(\vec{V}_{j \perp} \times \vec{\omega}_{c j}\right) \times \vec{\omega}_{c j} \quad(16)
\end{aligned}
$$

Since

So last equation becomes

$$
\left(\vec{V}_{j \perp} \times \vec{\omega}_{c j}\right) \times \vec{\omega}_{c j}=-\omega_{c j}^{2} \vec{V}_{j \perp}
$$

$$
\vec{V}_{j \perp}=\frac{i q_{j} \varphi_{1}\left[\vec{k}_{\perp} \times \vec{\omega}_{c j}-i \omega F_{j}^{\prime} \vec{k}_{\perp}\right]}{m_{j}\left[\omega^{2} F{ }_{j}^{2}-\omega_{c j}^{2}\right]}
$$

Taking parallel (II) component of equation (14), and putting $\quad \overrightarrow{\boldsymbol{V}}_{\boldsymbol{j} \|} \times \overrightarrow{\boldsymbol{\omega}}_{\boldsymbol{c} \boldsymbol{j}}=\mathbf{0}$

From equation (1),

$$
\begin{gathered}
\omega F^{\prime}{ }_{j} \vec{V}_{j \|}=\frac{q_{j} \vec{k}_{\|} \varphi_{1}}{m_{j}}+0 \\
\vec{V}_{j \|}=\frac{q_{j} \vec{k}_{\|} \varphi_{1}}{\omega F_{j}^{\prime} m_{j}}
\end{gathered}
$$

$$
\begin{gathered}
\frac{\partial n_{j 1}}{\partial t}=-n_{j o}\left(\vec{\nabla} \cdot \vec{V}_{j 1}\right) \\
-i \omega n_{j 1}=\frac{-n_{j o} i\left(\vec{k}_{\|}+\vec{k}_{\perp}\right)\left(\vec{V}_{j \|} \cdot \vec{V}_{j \perp}\right)}{n_{j 1}=\frac{n_{j o}}{\omega}\left(\vec{k}_{\|} \cdot \vec{V}_{j \|}+\vec{k}_{\perp} \cdot \vec{V}_{j \perp}\right)}
\end{gathered}
$$

Putting the values of $\overrightarrow{\boldsymbol{V}}_{\boldsymbol{j} \perp}$ and $\overrightarrow{\boldsymbol{V}}_{\boldsymbol{j} \|}$, we get

$$
\chi_{j}=\frac{k_{\perp}^{2}}{k^{2}} \frac{\omega_{p j}^{2} F_{j}^{\prime}}{\left(\omega_{c j}^{2}-\omega^{2} F_{j}^{2}\right)}-\frac{k_{\|}^{2}}{k^{2}} \frac{\omega_{p j}^{2}}{F_{\prime}^{\prime} \omega^{2}}
$$

Let us consider a super-cooled magnetized Fermi dusty plasma where

- Electrons are considered hot at Fermi temperature and quantum

- Ions are cold and non-quantum

- Dust particles are cold and non-quantum

Then

$$
\begin{aligned}
& \chi_{e}=\frac{k_{\perp}^{2}}{k^{2}} \frac{\omega_{p e}^{2} F_{e}^{\prime}}{\left(-\omega^{2} F_{e}^{2}\right)}-\frac{k_{\|}^{2}}{k^{2}} \frac{\omega_{p e}^{2}}{F_{e}^{\prime} \omega^{2}} \\
=- & \frac{k_{\perp}^{2}}{k^{2}} \frac{\omega_{p e}^{2}}{F_{e}^{\prime}}-\frac{k_{\|}^{2}}{k^{2}} \frac{\omega_{p e}^{2}}{F^{\prime} \omega_{e}^{2}} \\
= & -\frac{\left(k_{\perp}^{2}+k_{\|}^{2}\right)}{k^{2}} \frac{\omega_{p e}^{2}}{F_{e}^{\prime} \omega^{2}}
\end{aligned}
$$


Since $\boldsymbol{k}_{\perp}^{2}+\boldsymbol{k}_{\|}^{2}=\boldsymbol{k}^{2}$

Since $F_{e}^{\prime}=1-\frac{k^{2} V \prime_{F e}^{2}}{\omega^{2}}$

$$
\chi_{e}=-\frac{\omega_{p e}^{2}}{F_{e}^{\prime} \omega^{2}}
$$

$$
\chi_{e}=-\frac{\omega_{p e}^{2}}{\omega^{2}\left(1-\frac{k^{2} V_{F e}^{\prime 2}}{\omega^{2}}\right)}
$$

Assume $\omega^{2} \ll \boldsymbol{k}^{2} \boldsymbol{V}_{F e}^{\prime 2}$, and $\boldsymbol{F}_{e}^{\prime}=-\frac{\boldsymbol{k}^{2} V_{F e}^{2}}{\omega^{2}}$ then,

$$
\begin{aligned}
\chi_{e}=- & \frac{\omega_{p e}^{2}}{\omega^{2}\left(-\frac{k^{2} V_{F e}^{\prime 2}}{\omega^{2}}\right)} \\
= & \frac{\omega_{p e}^{2}}{k^{2} V_{F e}^{\prime 2}}
\end{aligned}
$$

Since $\lambda_{F e}^{\prime 2}=\frac{V_{F e}^{2}}{\omega_{p e}^{2}}$

$\lambda^{\prime}{ }_{F e}=$ Debye length of electrons at Fermi temperature

$\boldsymbol{V}_{\boldsymbol{F} \boldsymbol{e}}^{\prime}=$ Fermi speed of electrons

$\boldsymbol{\omega}_{\boldsymbol{p} \boldsymbol{e}}=$ electron plasma frequency

So,

$$
\chi_{e}=\frac{1}{k^{2} \lambda_{F e}^{\prime 2}}
$$

Now for $\chi_{i}, F^{\prime}{ }_{i}=\mathbf{1}$

by using $\boldsymbol{\omega}_{c i}^{2} \gg \boldsymbol{\omega}^{2}$

$$
\chi_{i}=\frac{K_{\perp}^{2}}{K^{2}} \frac{\omega_{p i}^{2}}{\omega_{c i}^{2}-\omega^{2}}-\frac{K_{\|}^{2}}{K^{2}} \frac{\omega_{p i}^{2}}{\omega^{2}}
$$

$$
\chi_{i}=\frac{K_{\perp}^{2}}{K^{2}} \frac{\omega_{p i}^{2}}{\omega_{c i}^{2}}-\frac{K_{\|}^{2}}{K^{2}} \frac{\omega_{p i}^{2}}{\omega^{2}}
$$

Similarly for $\chi_{\boldsymbol{d}}, \boldsymbol{F}_{\boldsymbol{d}}^{\prime}=\mathbf{1}$ and $\boldsymbol{\omega}_{\boldsymbol{c} \boldsymbol{d}}=\mathbf{0}$

So, the susceptibility for the un-magnetized and cold dust particles is obtained as

$$
\chi_{d}=-\frac{\omega_{p d}^{2}}{\omega^{2}}
$$

The dispersion relation for DLH wave in Quantum plasma will be

where

$$
\begin{gathered}
\varepsilon(\omega, \vec{K})=1+\frac{1}{k^{2} \lambda_{F e}^{\prime 2}}+\frac{k_{\perp}^{2} \omega_{p i}^{2}}{k^{2} \omega_{c i}^{2}}-\frac{k_{\|}^{2}}{k^{2}} \frac{\omega_{p i}^{2}}{\omega^{2}}-\frac{\omega_{p d}^{2}}{\omega^{2}}=0 \\
\frac{\omega_{p i}^{2}}{\omega_{c i}^{2}} \gg \frac{1}{\omega^{2} \lambda \prime_{F e}^{2}} \gg 1 \\
\omega^{2}=\frac{\omega_{d l h}^{2}\left(1+\frac{K_{\|}^{2} \omega_{p i}^{2}}{K^{2} \omega_{p d}^{2}}\right)}{\left.\frac{k_{\perp}^{2}\left(\frac{\omega_{c i}^{2}}{k^{2}} \cdot \frac{1}{c^{\prime}{ }_{F s}^{2}} \cdot 1\right)}{k_{\perp}^{2}}+1\right)}
\end{gathered}
$$


So

$$
\begin{aligned}
& \omega_{d l h}^{2}=\frac{\omega_{p d}^{2} \omega_{c i}^{2}}{\omega_{p i}^{2}} \quad, C_{F s}^{\prime 2}=\lambda_{F e}^{\prime 2} \omega_{p i}^{2} \text { and } \rho_{F s}^{\prime 2}=\frac{C_{F s}^{\prime 2}}{\omega_{c i}^{2}} \\
& \omega^{2}=\frac{\omega_{d l h}^{2}\left(1+\frac{K_{\|}^{2} \omega_{p i}^{2}}{K^{2} \omega_{p d}^{2}}\right)}{\frac{k_{\perp}^{2}}{k^{2}}\left(\frac{1}{k_{\perp}^{2}} \cdot \frac{1}{\rho^{\prime 2}}+1\right)}
\end{aligned}
$$

Since

Therefore

$$
\begin{gathered}
k_{\perp}^{2} \gg k_{\|}^{2} \\
k_{\perp}^{2}+k_{\|}^{2}=k^{2} \\
k_{\perp}^{2} \sim k^{2} \Rightarrow \frac{k_{\perp}^{2}}{k^{2}}=1
\end{gathered}
$$

Or

$$
\begin{aligned}
& \omega^{2}=\omega_{d l h}^{2}\left(1+\frac{K_{\|}^{2}}{K^{2}} \frac{\omega_{p i}^{2}}{\omega_{p d}^{2}}\right) \times\left(1+\frac{1}{K_{\perp}^{2} \rho_{F S}^{\prime 2}}\right)^{-1} \\
& \omega^{2}=\omega_{d l h}^{2}\left(1+\frac{K_{\|}^{2}}{K^{2}} \frac{\omega_{p i}^{2}}{\omega_{p d}^{2}}\right) \times\left(1-\frac{1}{K_{\perp}^{2} \rho_{F S}^{\prime 2}}\right)
\end{aligned}
$$

is the dispersion relation of the DLH wave in the cold Fermi dusty magneto plasma.

As

$$
\lambda_{F e}^{\prime 2}=\frac{V_{F e}^{2}\left(1+\gamma_{e}\right)}{\omega_{p e}^{2}}
$$

So, the relation for $\boldsymbol{C}_{F S}^{\prime 2}$ is

$$
\begin{gathered}
C_{F s}^{\prime 2}=\frac{\omega_{p i}^{2}}{\omega_{p e}^{2}} V_{p e}^{2}\left(1+\gamma_{e}\right) \\
C_{F s}^{\prime 2}=\frac{\omega_{p i}^{2}}{\omega_{p e}^{2}} \cdot \frac{2 K_{B} T_{F e}}{m_{e}}\left(1+\frac{\hbar^{2} K^{2}}{8 m_{e} K_{B} T_{F e}}\right) \\
C_{F s}^{\prime 2}=\frac{2 K_{B} T_{F e}}{m_{i}}\left(1+\frac{\hbar^{2} K^{2}}{8 m_{e} K_{B} T_{F e}}\right)
\end{gathered}
$$

\section{Results and Discussion}

- $\boldsymbol{\rho}_{F \boldsymbol{s}}^{\prime}$ is the ion gyro radius at the electron Fermi temperature with quantum correction as

$$
\gamma_{e}=\frac{\hbar^{2} k^{2}}{8 m_{e} k_{B} T_{F e}}
$$

- The DLH wave is seen to be significantly modified by the quantum effect.

- In this case electrons at the Fermi temperature drive the wave.

- If the electrons and ions within the plasma have different temperatures such as $\boldsymbol{T}_{\boldsymbol{e}} \gg \boldsymbol{T}_{\boldsymbol{i}}$ then there is a sort of hybrid wave that depends upon the ion mass 
$m_{i}$ and Fermi temperature of electron $\boldsymbol{T}_{\boldsymbol{F} \boldsymbol{e}}$.

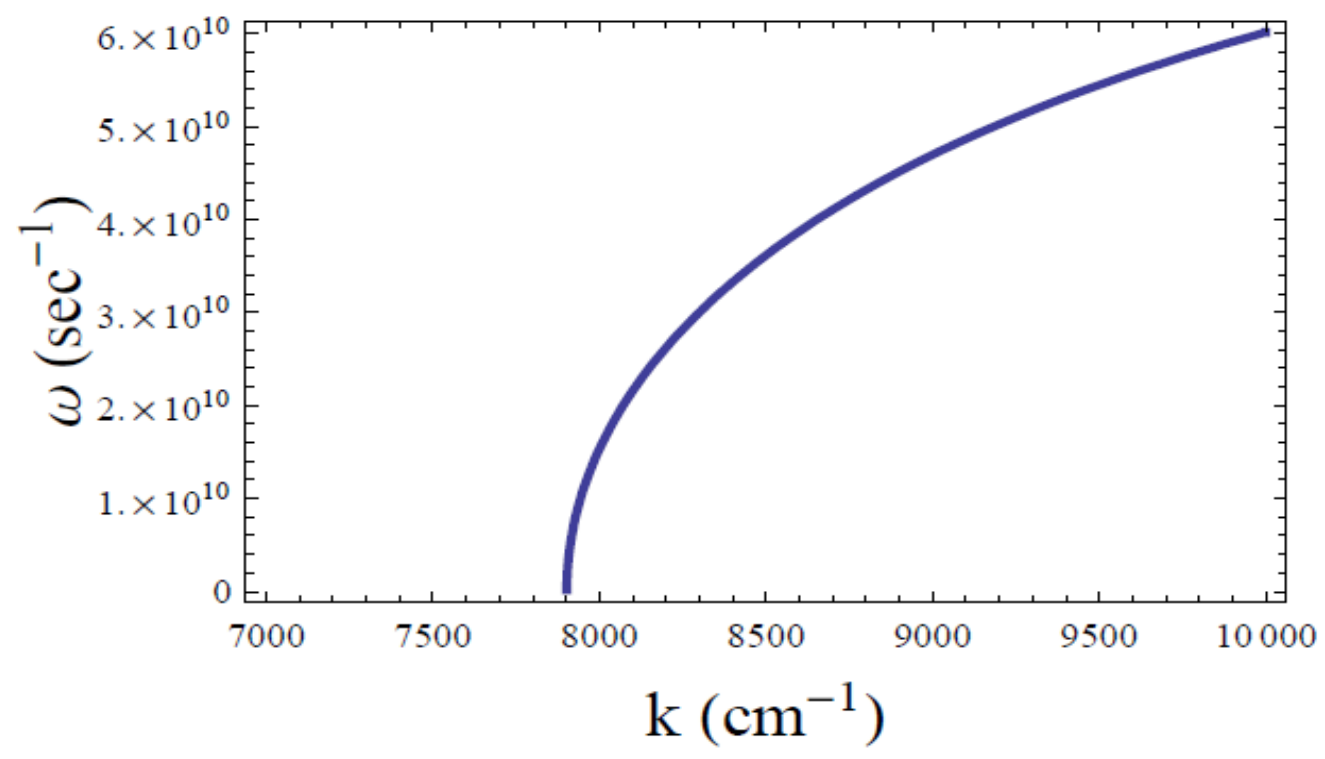

Figure 1. Variation of $\omega$ as a function of wave number $\mathrm{k}$

Figure 1 shows that frequency of the quantum dust-lower-hybrid wave increases with the increase of $\mathrm{k}$.

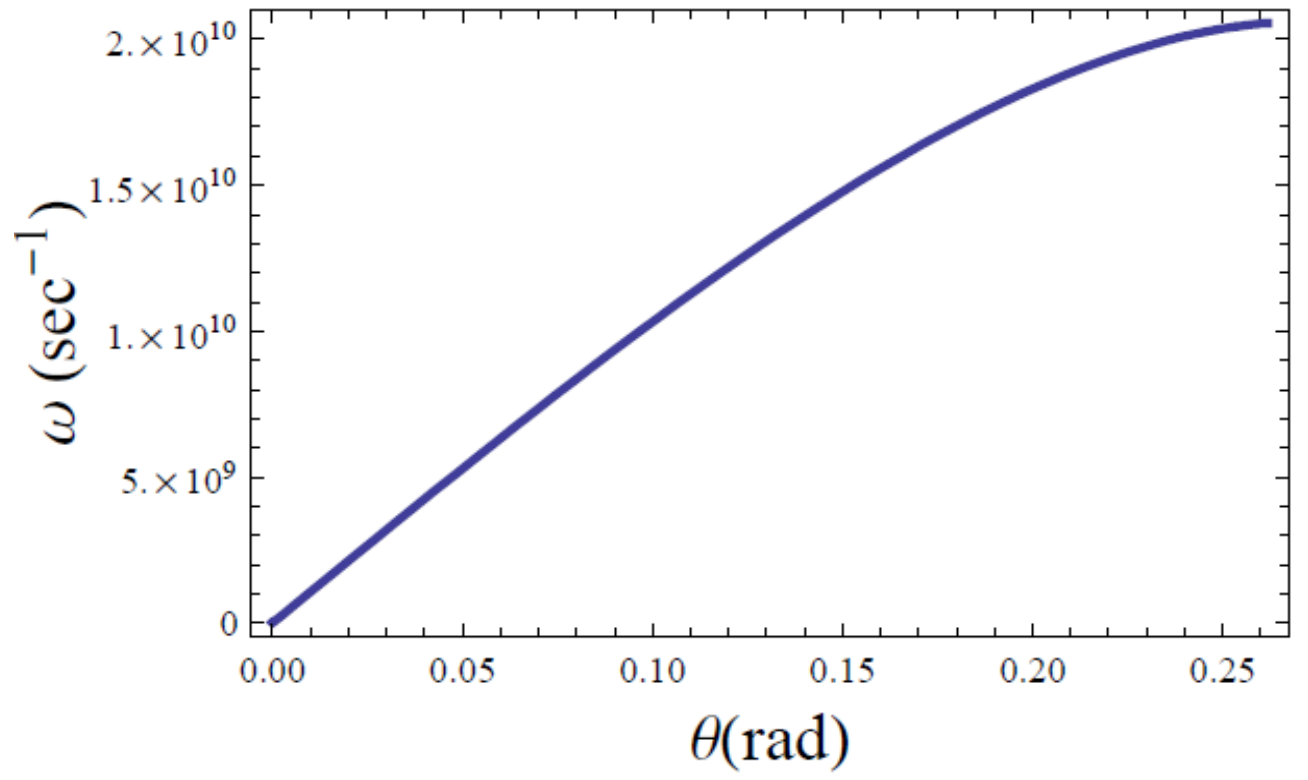

Figure 2. Variation of $\omega$ as a function of propagation angle $\theta$

Figure 2 shows that frequency of the quantum dust-lower-hybrid wave increases at small angle of propagation. 


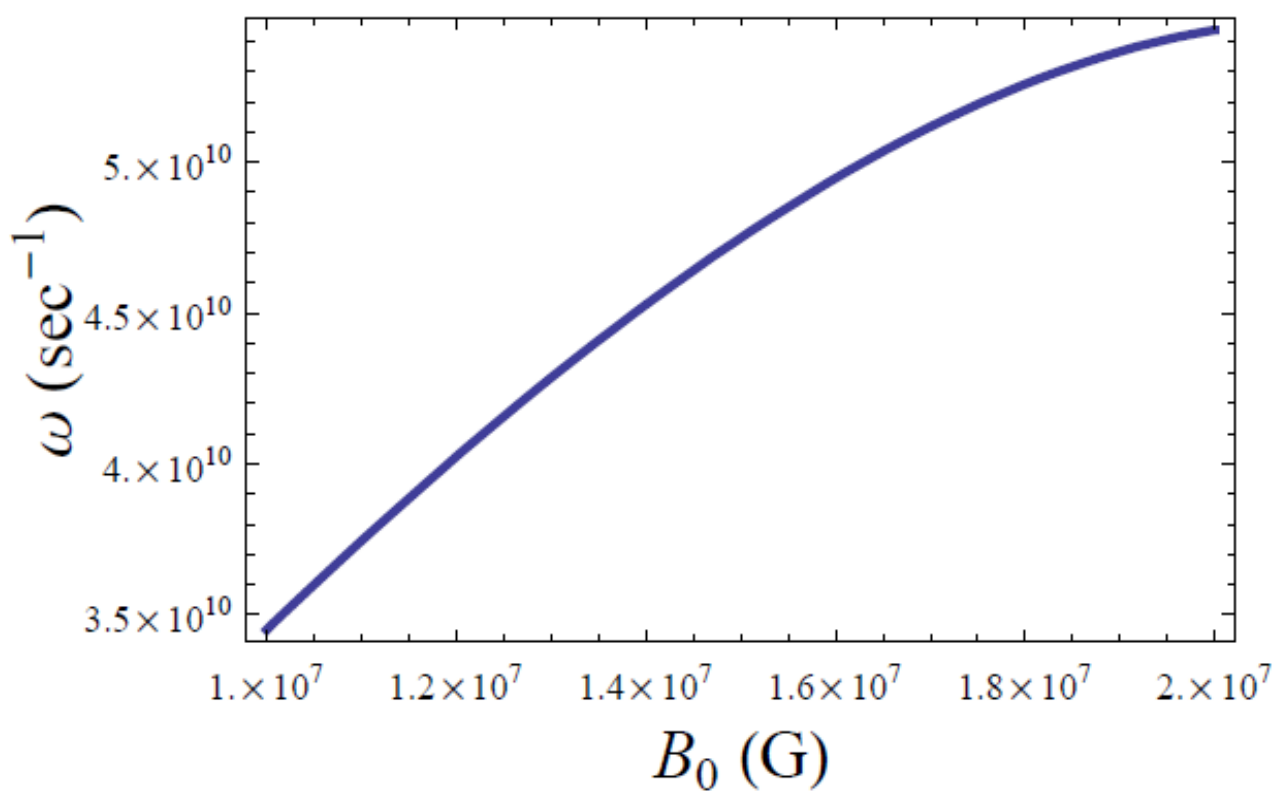

Figure 3. Variation of $\omega$ as a function of external magnetic field $B_{0}$

and figure 3 shows that frequency of the quantum dust-lower-hybrid wave increases with the increase of magnetic field $B_{0}$.

\section{Conclusion}

Equation (25) is the dispersion relation of the DLH wave in an ultra-cold and uniformly magnetized Fermi dusty plasma employing the quantum hydrodynamic model of a plasma with quantum and thermal corrections.

This relation is significantly affected by the quantum correction. This will find applications in diagnosing the charged dust impurities in microelectronics and wave particle interactions in the dusty quantum magnetoplasma. Bohm potential term shows how the concept of quantum potential leads to the notion of an "unbroken wholeness of entire universe".

\section{References}

[1] Haas F, Garcia LG, Goedert,J, Manfredi,G. Quantum ion-acoustic waves. Phys. Plasmas. 2003;10(10): 3858.

[2]Markowich PA, Ringhofer CA, Schmeiser C. Semiconductor Equations. Vienna: Springer; 1990.

[3] Kremp D, Bornath TH, Bonitz M, Schlanges M. Quantum kinetic theory of plasmas in strong laser fields. Phys. Rev. 1999;E 60; 4725. 
[4] Jung YD. Quantum-mechanical effects on electron-electron scattering in dense high-temperature plasmas. Phys Plasmas. 2001;8: 3842.

[5] Opher M, Silva LO, Dauger DE, Decyk VK, Dawson JM. Quantum ionacoustic waves. Phys Plasma. 2001;8: 2454.

[6] Chabrier G, Douchin F, Potekhin AY. Dense astrophysical plasmas. J Phys: Condens Matter. 2002;14: 9133.

[7] Shukla PK. A new dust mode in quantum plasmas. Phys Lett A. 2006;352(3): 242-243.

[8] Shukla PK, Stenflo L. Jeans instabilities in quantum dusty plasmas. Phys Lett A. 2006(4-5):355: 378-380.

[9] Shukla PK, Ali S. Dust acoustic waves in quantum plasmas. Phys Plasma. 2005;12(11): 114502.

[10] Shukla PK, Stenflo L, Bingham R. Shielding of a slowly moving test charge in a quantum plasmas. Phys Lett A. 2006;359(3): 218-219. 